\title{
Effect of dexmedetomidine on the cognitive function of patients undergoing gastric cancer surgery by regulating the PI3K/AKT signaling pathway
}

\author{
ZHIYUAN WANG, ZIJIN SHEN, HAIBIN WANG, LIN ZHANG and RONG DONG \\ Department of Anesthesiology, Ruijin Hospital North, Shanghai Jiaotong \\ University School of Medicine, Shanghai 201801, P.R. China
}

Received September 2, 2019; Accepted October 30, 2019

DOI: $10.3892 / \mathrm{ol} .2019 .11224$

\begin{abstract}
Effect of dexmedetomidine on the cognitive function of patients undergoing gastric cancer surgery by regulating the phosphoinositide 3-kinase (PI3K)/AKT signaling pathway was investigated. A total of 110 patients who were diagnosed and underwent radical gastrectomy in Ruijin Hospital North, Shanghai Jiaotong University School of Medicine from July 2016 to July 2018 were selected. In the experimental group, 60 patients were treated with dexmedetomidine infusion. In the control group, 50 patients were injected with $0.9 \%$ sodium chloride injection during the same period. The expression levels of serum inflammatory factors tumor necrosis factor- $\alpha$ (TNF- $\alpha$ ), interleukin-6 (IL-6), PI3K and AKT of patients were compared between the two groups before and after surgery for 1 day. The number of adverse reactions in the two groups was compared. The correlation between mini-mental state examination (MMSE) score and the expression levels of serum IL-6, PI3K and AKT was compared. The levels of serum TNF- $\alpha$, IL-6, PI3K and AKT after operation for 1 day of patients in the two groups were significantly higher than those before operation $(\mathrm{P}<0.05)$, and were lower in the experimental than in the control group $(\mathrm{P}<0.05)$. The number of postoperative cognitive dysfunction of patients in the experimental group was lower than that of patients in the control group $(\mathrm{P}<0.05)$. The total number of adverse reactions in the control group was higher than that of patients in the experimental group $(\mathrm{P}<0.05)$. The MMSE scores of the two groups were decreased at 1 day after operation and were significantly lower in the control group than in the experimental group $(\mathrm{P}<0.05)$. The MMSE score was negatively correlated with the expression levels of serum
\end{abstract}

Correspondence to: Dr Rong Dong, Department of Anesthesiology, Ruijin Hospital North, Shanghai Jiaotong University School of Medicine, 999 Xiwang Road, Shanghai 201801, P.R. China E-mail: r5p5ys@163.com

Key words: dexmedetomidine, PI3K/AKT, tumor necrosis factor- $\alpha$, interleukin-6, cognitive function
TNF- $\alpha$, IL-6, PI3K and AKT $(\mathrm{P}<0.001)$. Dexmedetomidine can effectively reduce the expression levels of postoperative inflammatory factors in patients undergoing gastric cancer surgery, improve the postoperative cognitive function by regulating PI3K-Akt signaling pathway and promotes the recovery of postoperative cognitive function.

\section{Introduction}

Gastric cancer is one of the deadly malignant tumors (1). During surgical procedures, inflammatory factor-mediated cascade reaction may trigger cerebral ischemia and hypoxia. Subsequent destruction of the blood-brain barrier leads to neuronal apoptosis and postoperative cognitive dysfunction (POCD) (2), reduces the quality of life of patients and affects the prognosis of patients.

Studies have shown that (3) phosphoinositide 3-kinase (PI3K) signal transduction pathway is one of the most frequently altered pathways in human cancer and plays a crucial role in the formation and deterioration of tumors. The PI3K/Akt pathway is involved in cognitive impairment caused by ischemia-reperfusion injury (4). Studies have also shown that the changes of cell signaling play a crucial role in the deterioration of gastric cancer (5). In the deterioration of gastric cancer, it has been reported that PI3K/Akt signal transduction pathway is one of the pathways of maladjustment, so the PI3K/Akt signaling pathway plays a crucial role in gastric cancer (6).

Dexmedetomidine is an $\alpha 2$-adrenoceptor agonists with sedative analgesic, anxiolytic activity and inhibition of sympathetic excitation (7). The most important feature of dexmedetomidine is that patients can be easily awakened from sedation (8). In addition, due to the low effect of dexmedetomidine on breathing, it has become the preferred sedative in many operations, such as conscious craniotomy and conscious sedation (9).

Studies have shown that dexmedetomidine has a positive effect on the protection of postoperative cognitive function (10). However, there are few studies on whether dexmedetomidine influences the cognitive function of patients undergoing gastric cancer surgery through the PI3K-Akt pathway, thus this aspect was studied. 


\section{Patients and methods}

Baseline data. A total of 100 patients were diagnosed and underwent radical gastrectomy in Ruijin Hospital North, Shanghai Jiaotong University School of Medicine (Shanghai, China) from July 2016 to July 2018. In experimental group, 60 patients were treated with dexmedetomidine infusion. There were 38 males and 22 females with an average age of $68.37 \pm 3.27$ years. In control group, 50 patients were injected with the same amount and concentration of $0.9 \%$ sodium chloride injection during the same period. There were 30 males and 20 females, with an average age of $68.26 \pm 2.07$ years. There were no significant differences between the two groups in age and sex.

\section{Inclusion and exclusion criteria}

Inclusion criteria. Diagnosed with gastric cancer and consented to surgical resection; no radiotherapy, chemotherapy, or comorbidity with other malignant tumors.

Exclusion criteria. Patients with liver and renal dysfunction, communication and cognitive dysfunction; patients who did not cooperate with the requirements of the study.

This study was approved by the Ethics Committee of Ruijin Hospital North, Shanghai Jiaotong University School of Medicine. Patients and their families agreed to the participation in this experiment and signed an informed consent form.

\section{Experimental methods}

Surgical methods. Before the induction of anesthesia, patients in the experimental group received dexmedetomidine (China National Medicines Guorui Pharmaceutical Co.,Ltd.) infusion: The initial dose was intravenous drip of $0.5 \mu \mathrm{g} / \mathrm{kg}-1$ and the maintenance dose was intravenous injection of $0.4 \mu \mathrm{g} / \mathrm{kg}-1 \mathrm{~h}$ -1 . In the control group, patients were injected with the same amount and concentration of $0.9 \%$ sodium chloride during the same period. All patients underwent radical gastrectomy under general anesthesia. In the two groups, patients were intravenously injected with midazolam of $0.1 \mathrm{mg} / \mathrm{kg}$, propofol of $1.5 \mathrm{mg} / \mathrm{kg}$, sufentanil of $0.4 \mu \mathrm{g} / \mathrm{kg}$ and vecuronium of $1.0 \mathrm{mg} / \mathrm{kg}$ in turn. Anesthesia was maintained with propofol + sevoflurane + vecuronium during surgery. Sufentanil was added or not according to need of the operation.

Detection methods. In the morning fasting state, $5 \mathrm{ml}$ of the patient's elbow venous blood was taken $24 \mathrm{~h}$ before and after surgery and centrifuged at $1,500 \mathrm{x}$ a at $4^{\circ} \mathrm{C}$ for $10 \mathrm{~min}$. The supernatant was placed in a refrigerator at $-80^{\circ} \mathrm{C}$ for later use. The expression levels of tumor necrosis factor (TNF- $\alpha$ ), S-100 $\beta$ protein, interleukin (IL-6), PI3K and Akt in the serum of patients in the two groups were detected by enzyme-linked immunosorbent assay (ELISA). All the kits were provided by Shanghai Guangrui Biotechnology Co., Ltd. Specific operations were carried out strictly in accordance with the instructions of the kit.

Observation indexes. (i) The expression levels of serum inflammatory factors TNF- $\alpha$ and IL- 6 of patients in the two groups before and after operation for 1 day; (ii) the expression levels of serum PI $3 \mathrm{~K}$ and AKT of patients in the two groups before and after surgery for 1 day; (iii) the number of adverse reactions in the two groups was compared: POCD, hypotension and bradycardia. The criterion for hypotension was systolic blood pressure $<90 \mathrm{mmHg}$; the criterion for sinus-ventricular bradycardia was heart rate $<55$ times per minute; (iv) the minimental state examination (MMSE) scores of patients were compared between the two groups: The MMSE score was used to assess the patient's cognitive ability. Postoperative MMSE score decreased by 2 points or more than preoperative MMSE score, which was judged as POCD and (v) the correlation between MMSE score and the expression levels of serum IL-6, PI3K and AKT.

Statistical analysis. Statistical analysis was performed by using SPSS 20.0. (IBM Corp., Armonk, NY, USA) The measurement data were expressed as mean number \pm standard deviation (mean $\pm \mathrm{SD}$ ). The $\mathrm{t}$-test was used for comparison. The paired t-test was used for comparison between groups. Enumeration data were expressed as $\mathrm{n} / \% . \chi^{2}$ test was used for comparison. The difference was statistically significant at $\mathrm{P}<0.05$.

\section{Results}

Comparison of baseline data between the two groups. There was no significant difference in baseline data of age, BMI or TNM stages between the two groups $(\mathrm{P}>0.05)$ (Table I).

The expression levels of serum inflammatory factors of patients in the two groups before and after operation for 1 day. There were no significant differences in preoperative TNF- $\alpha$ and IL- 6 levels of patients between the two groups $(\mathrm{P}>0.05)$. Serum TNF- $\alpha$ and IL-6 levels of patients in both groups after surgery for 1 day were significantly higher than those before surgery $(\mathrm{P}<0.05)$. The levels of serum TNF- $\alpha$ and IL-6 in the experimental group after surgery for 1 day were lower than those in the control group $(\mathrm{P}<0.05)$ (Fig. 1).

The expression levels of serum PI3K and AKT of patients in the two groups before and after operation for 1 day. There were no significant differences in preoperative PI3K and AKT levels of patients between the two groups $(P>0.05)$. Serum $\mathrm{PI} 3 \mathrm{~K}$ and AKT levels of patients in both groups after surgery for 1 day were significantly higher than those before surgery $(\mathrm{P}<0.05)$. The levels of serum PI3K and AKT in the experimental group after surgery for 1 day were lower than those in the control group $(\mathrm{P}<0.05)$ (Fig. 2).

The number of adverse reactions of patients in both groups. The number of POCD of patients in the experimental group was lower than that of patients in the control group $(\mathrm{P}<0.05)$. There was no significant difference between the two groups in the number of hypotension and bradycardia $(P>0.05)$, but the total number of adverse reactions in the control group was higher than that of patients in the experiment group. The difference was statistically significant $(\mathrm{P}<0.05)$ (Table II).

The MMSE score of patients in the two groups before and after operation for 1 day. There was no significant difference in preoperative MMSE scores of patients between the two groups $(\mathrm{P}>0.05)$. The MMSE scores of the two groups were 
Table I. Comparison of baseline data between the two groups (n/\%).

\begin{tabular}{|c|c|c|c|c|}
\hline Factors & $\begin{array}{l}\text { Experimental group } \\
\qquad(n=60)\end{array}$ & $\begin{array}{l}\text { Control group } \\
\quad(n=50)\end{array}$ & $t / \chi^{2}$ & P-value \\
\hline Age/years & $68.37 \pm 3.27$ & $68.26 \pm 2.07$ & 0.206 & 0.837 \\
\hline BMI $\left(\mathrm{kg} / \mathrm{m}^{2}\right)$ & $21.3 \pm 2.45$ & $21.5 \pm 2.36$ & 0.433 & 0.666 \\
\hline \multicolumn{5}{|l|}{ TNM stages } \\
\hline Stage I & $28(46.67)$ & $21(42)$ & & \\
\hline Stage II & $32(53.33)$ & $29(58)$ & 0.240 & 0.624 \\
\hline \multicolumn{5}{|l|}{ Gender } \\
\hline Male & $38(63.33)$ & $30(60)$ & & \\
\hline Female & $22(36.67)$ & $20(40)$ & 0.128 & 0.720 \\
\hline \multicolumn{5}{|l|}{ Hypertension } \\
\hline Yes & $20(33.33)$ & $18(36)$ & & \\
\hline No & $40(66.67)$ & $32(64)$ & 0.086 & 0.770 \\
\hline \multicolumn{5}{|c|}{ Diabetes mellitus } \\
\hline Yes & $26(43.33)$ & $22(44)$ & & \\
\hline No & $34(56.67)$ & $28(56)$ & 0.005 & 0.944 \\
\hline \multicolumn{5}{|c|}{ Hyperlipidemia } \\
\hline Yes & $22(36.67)$ & $23(46)$ & & \\
\hline No & $38(63.33)$ & $27(54)$ & 0.983 & 0.322 \\
\hline \multicolumn{5}{|l|}{ Smoking } \\
\hline Yes & 43 (71.67) & $34(68)$ & & \\
\hline No & $17(28.33)$ & $16(32)$ & 0.175 & 0.676 \\
\hline \multicolumn{5}{|l|}{ Drinking } \\
\hline Yes & $34(56.67)$ & $35(70)$ & & \\
\hline No & $26(43.33)$ & $15(30)$ & 2.074 & 0.149 \\
\hline
\end{tabular}

Table II. The number of adverse reactions of patients in both groups $(\mathrm{n} / \%)$.

\begin{tabular}{lcccc}
\hline & $\begin{array}{c}\text { Experimental } \\
\text { group } \\
(\mathrm{n}=60)\end{array}$ & $\begin{array}{c}\text { Control } \\
\text { group } \\
(\mathrm{n}=50)\end{array}$ & $\chi^{2}$ & P-value \\
Grouping & $12(20)$ & $20(40)$ & 5.288 & 0.022 \\
POCD & $5(8.33)$ & $6(12)$ & 0.901 & 0.343 \\
Hypotension & $4(6.67)$ & $3(6)$ & 0.020 & 0.887 \\
Bradycardia & $21(35)$ & $29(58)$ & 5.819 & 0.016 \\
Total number & & &
\end{tabular}

POCD, postoperative cognitive dysfunction.

decreased at 1 day after operation and the MMSE scores of patients in the control group were significantly lower than those of patients in the experimental group. The difference was statistically significant $(\mathrm{P}<0.05)$ (Table III).

Correlation analysis between MMSE score and the expression levels of serum IL-6,TNF- $\alpha, P I 3 K$ and AKT. The results of correlation analysis showed that the MMSE score was negatively correlated with the expression levels of serum inflammatory factors TNF- $\alpha$ and IL-6 $(\mathrm{P}<0.001)$ and
Table III. The MMSE score of patients in the two groups.

\begin{tabular}{lcccc}
\hline & $\begin{array}{c}\text { Experimental } \\
\text { group } \\
(\mathrm{n}=60)\end{array}$ & $\begin{array}{c}\text { Control } \\
\text { group } \\
(\mathrm{n}=50)\end{array}$ & t-test & P-value \\
\hline $\begin{array}{l}\text { Before } \\
\text { operation } \\
\begin{array}{l}\text { After operation } \\
\text { for 1 day }\end{array}\end{array}$ & $28.54 \pm 4.34$ & $28.48 \pm 4.25$ & 0.073 & 0.942 \\
\hline MMSE, mini-mental state examination. & & & & \\
\hline
\end{tabular}

negatively correlated with the expression levels of PI3K and AKT $(\mathrm{P}<0.001)$ (Fig. 3).

\section{Discussion}

The clinical manifestations of POCD includes insanity, anxiety, personality changes and memory loss. It may be related to anesthesiamethods,typesofsurgery,complicationsandpotential cognitive differences (11). Therefore, it is very important to study whether the use of dexmedetomidine anesthetic in 

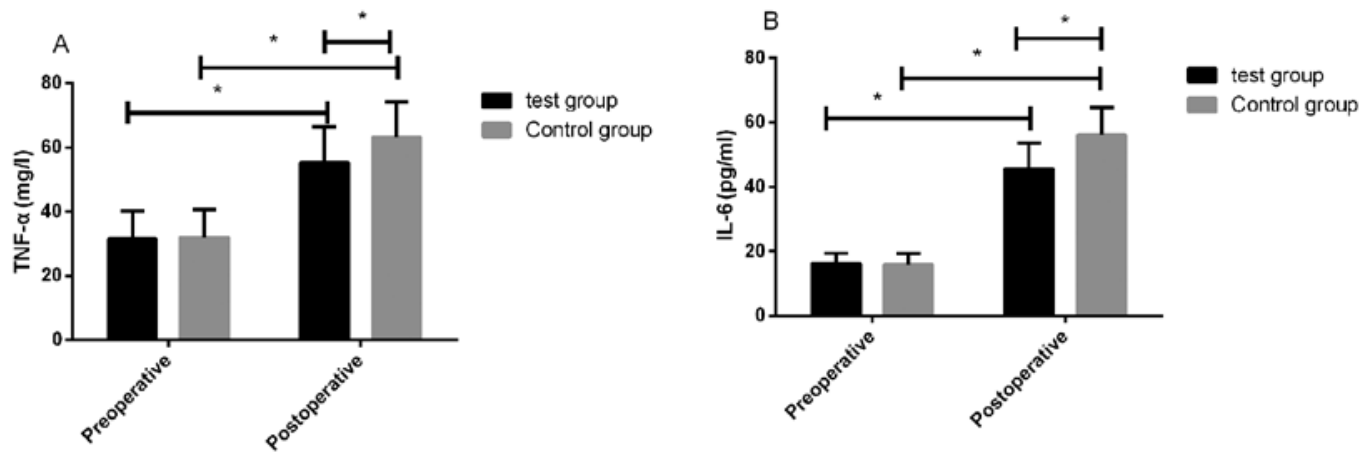

Figure 1. Expression levels of serum TNF- $\alpha$ and IL- 6 of patients in the two groups before and after operation for 1 day. (A) Serum TNF- $\alpha$ levels of patients in both groups after surgery for 1 day were significantly higher than those before surgery. The levels of serum TNF- $\alpha$ of patients in the experimental group were lower than those of patients in the control group. (B) Serum IL-6 levels of patients in both groups after surgery for 1 day were significantly higher than those before surgery. The levels of serum IL- 6 of patients in the experimental group after surgery were lower than those in the control group. "P<0.05. TNF- $\alpha$, tumor necrosis factor- $\alpha$, IL-6, interleukin- 6 .
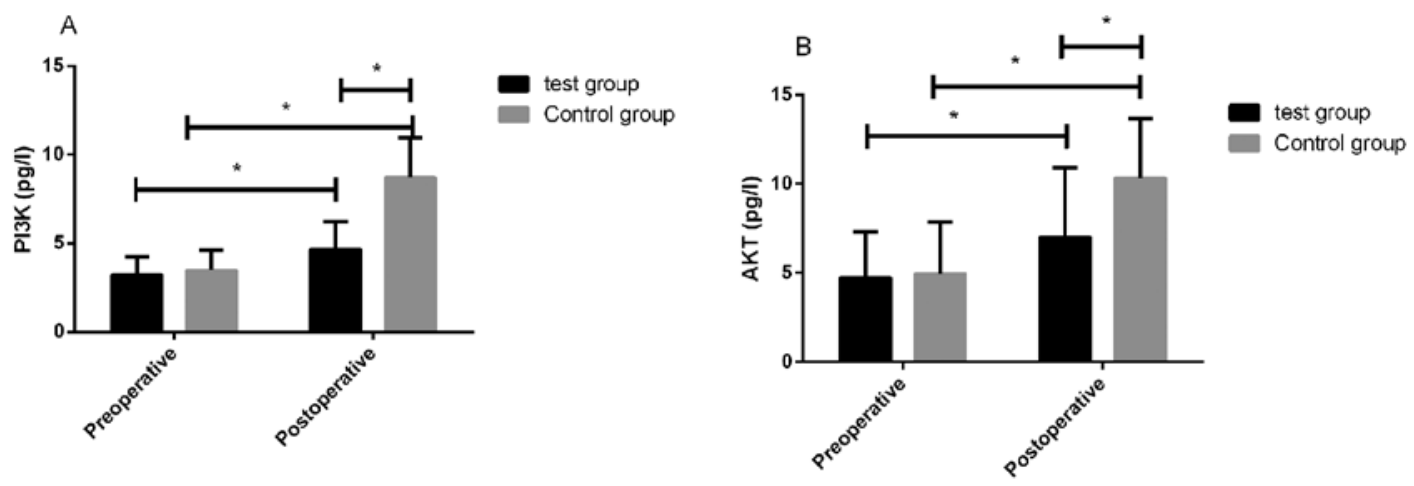

Figure 2. Expression levels of serum PI3K and AKT of patients in the two groups before and after operation for 1 day. (A) Serum PI3K levels of patients in both groups after surgery for 1 day were significantly higher than those before surgery. The levels of serum PI3K of patients in the experimental group were lower than those of patients in the control group. (B) Serum AKT levels of patients in both groups after surgery for 1 day were significantly higher than those before surgery. The levels of serum AKT of patients in the experimental group were lower than those of patients in the control group. " $\mathrm{P}<0.05$.
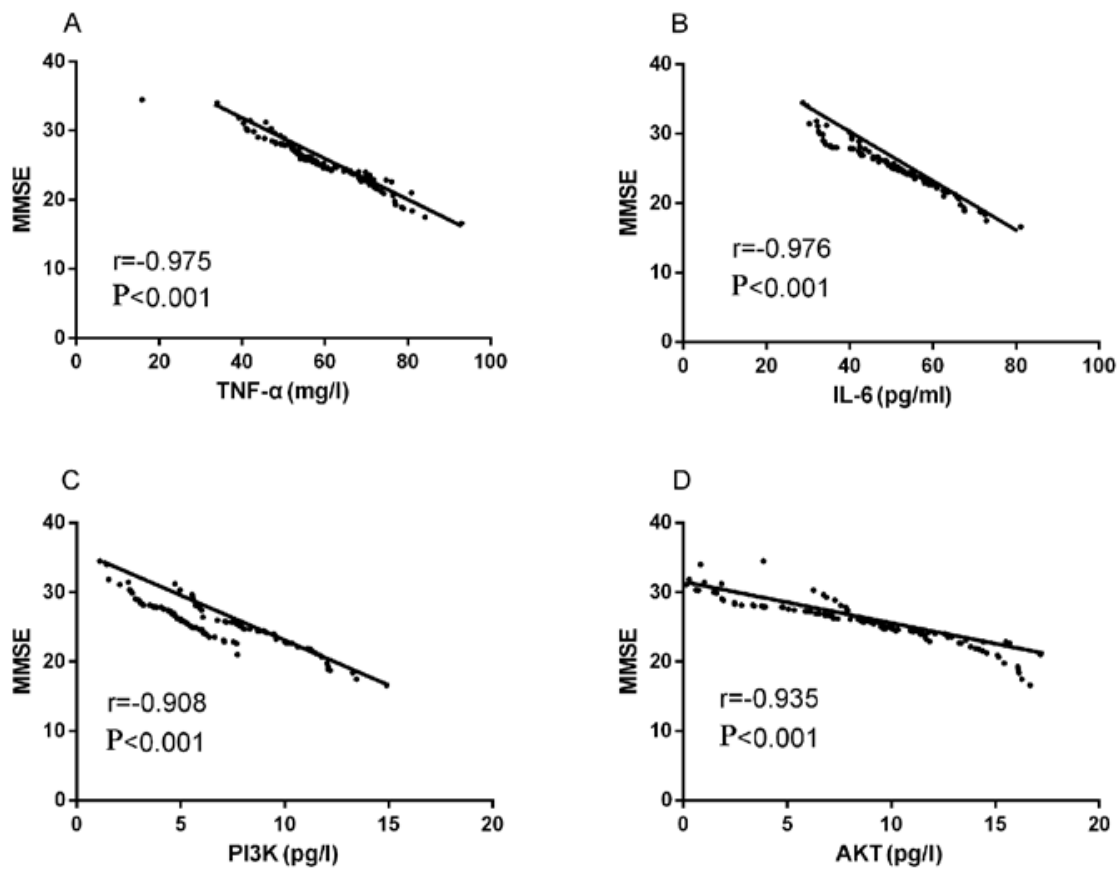

Figure 3. Correlation analysis between MMSE score and expression levels of serum IL-6, TNF- $\alpha$, PI3K and AKT. (A) MMSE score was negatively correlated with the expression levels of serum TNF- $\alpha(r=-0.975, P<0.001)$. (B) MMSE score was negatively correlated with the expression levels of serum IL-6 ( $r=-0.976$, $\mathrm{P}<0.001)$. (C) MMSE score was negatively correlated with the expression levels of serum PI3K $(\mathrm{r}=-0.908, \mathrm{P}<0.001)$. (D) MMSE score was negatively correlated with the expression levels of serum AKT ( $\mathrm{r}=-0.935, \mathrm{P}<0.001)$. MMSE, mini-mental state examination; TNF- $\alpha$, tumor necrosis factor- $\alpha$, IL-6, interleukin-6. 
gastric cancer surgery would cause POCD complications in patients. Studies have shown that dexmedetomidine, as a neurosurgical anesthetic, has a unique sedative and analgesic effect, does not cause respiratory depression, has stable hemodynamics and neuroprotective effects on postoperative bleeding and promote the recovery of nerve function (12). The PI3K/AKT signaling pathway plays an important role in the study of anesthetic drugs, neuronal damage and POCD mechanisms (13). However, it is unclear whether dexmedetomidine has an effect on the cognitive function of patients undergoing gastric cancer surgery through the PI3K/ AKT signaling pathway.

TNF- $\alpha$ and IL- 6 have important biological functions in immune and inflammatory responses. Normal levels of IL-6 are associated with the protection and repair of neurons. Conversely, higher levels of IL- 6 will increase neuronal damage (14). Studies have shown that TNF- $\alpha$ may not only participate in the process of memory, but also play a certain role in the pathophysiological process of POCD (15). The results of this study showed that there was no statistical difference in the expression levels of TNF- $\alpha$ and IL- 6 of patients between the two groups before operation. The serum TNF- $\alpha$ and IL- 6 levels of patients after operation for 1 day were significantly higher than those before surgery, but the levels of serum TNF- $\alpha$ and IL- 6 of patients in the experimental group after surgery for 1 day were lower than those in the control group. It showed that dexmedetomidine can inhibit the release of inflammatory mediators of TNF- $\alpha$ and IL-6. Studies have also confirmed that dexmedetomidine can inhibit the release of various inflammatory factors such as IL-6 and TNF- $\alpha$ in endotoxin shock rats (16) and this is consistent with the results of our present study.

The PI3K/Akt signaling pathway plays a key role not only in cell proliferation and apoptosis, but also plays an important role in regulating the body's inflammatory response (17). Studies have shown that dexmedetomidine improves neurocognitive damage caused by repeated propofol stimulation in rats. This is partly due to its anti-apoptotic effect and normalization of the destruction of PI3K/Akt signal transduction pathway (18). Our results showed that there was no significant difference in preoperative PI3K and AKT levels of patients between the two groups. Serum PI3K and AKT levels of patients in both groups after surgery for 1 day were significantly higher than those before surgery. The levels of serum PI3K and AKT in the experimental group after surgery for 1 day were lower than those in the control group. It indicated that dexmedetomidine can effectively reduce the levels of $\mathrm{PI} 3 \mathrm{~K}$ and $\mathrm{AKT}$ of patients in serum. Dexmedetomidine participates in the role of PI3K/Akt pathway in patients. Studies have shown that PI3K/Akt signaling pathway is involved in the process of dexmedetomidine to alleviate brain and kidney ischemic injury caused by reperfusion in rats (19). The results of Zhu et al (20) showed that dexmedetomidine can alleviate cerebral ischemia-reperfusion injury through PI3K/Akt signaling pathway, which is consistent with our findings.

Subsequently, we found that the number of POCD of patients in the experimental group was lower than that of patients in the control group and the MMSE scores of patients in the control group were significantly lower than those of patients in the experimental group, indicating that dexmedetomidine has a positive effect on improving MMSE score, reducing POCD and decreasing postoperative adverse reactions. The results are consistent with those of Qian et al (21). The results of correlation analysis showed that the MMSE score was negatively correlated with the expression levels of serum inflammatory factors TNF- $\alpha$ and IL-6 and negatively correlated with the expression levels of PI3K and AKT. Dexmedetomidine can reduce the expression levels of serum inflammatory factors TNF- $\alpha$, IL-6, PI3K and AKT improve MMSE scores. Postoperative cognitive dysfunction is also related to the levels of inflammatory factors (22). It is speculated that dexmedetomidine may reduce POCD through inflammatory factors TNF- $\alpha$ and IL- 6 mediated PI3K/AKT signaling pathway, but this also requires further certification in subsequent studies.

In conclusion, dexmedetomidine can effectively reduce the expression levels of postoperative inflammatory factors in patients undergoing gastric cancer surgery, improve the postoperative cognitive function by regulating PI3K-Akt signaling pathway and promote the recovery of postoperative cognitive function.

\section{Acknowledgements}

Not applicable.

\section{Funding}

This study was supported by Application of Full-depth Anesthesia Monitor in Postoperative Analgesia and Cognitive Function Evaluation of Flurbiprofen Ester in Elderly Patients of Youth Scientific Research Project, Jiading Health and Family Planning Commission of Shanghai (No. 2017-QN-01).

\section{Availability of data and materials}

The datasets used and/or analyzed during the present study are available from the corresponding author on reasonable request.

\section{Authors' contributions}

ZW, ZS and HW conceived and designed the study, and drafted the manuscript. ZW, ZS, LZ and RD collected, analyzed and interpreted the experimental data. $\mathrm{RD}$ revised the manuscript for important intellectual content. All authors read and approved the final manuscript.

\section{Ethics approval and consent to participate}

This study was approved by the Ethics Committee of Ruijin Hospital North, Shanghai Jiaotong University School of Medicine (Shanghai, China). Patients and their families agreed to the participation in this experiment and signed an informed consent form.

\section{Patient consent for publication}

Not applicable. 


\section{Competing interests}

The authors declare that they have no competing interests.

\section{References}

1. Jemal A, Bray F, Center MM, Ferlay J, Ward E and Forman D: Global cancer statistics. CA Cancer J Clin 61: 69-90, 2011.

2. Hudetz JA, Patterson KM, Iqbal Z, Gandhi SD and Pagel PS: Remote ischemic preconditioning prevents deterioration of short-term postoperative cognitive function after cardiac surgery using cardiopulmonary bypass: Results of a pilot investigation. J Cardiothorac Vasc Anesth 29: 382-388, 2015.

3. Lien EC, Dibble CC and Toker A: PI3K signaling in cancer: Beyond AKT. Curr Opin Cell Biol 45: 62-71, 2017.

4. Zhou S, Fang Z, Wang G and Wu S: Gap junctional intercellular communication dysfunction mediates the cognitive impairment induced by cerebral ischemia-reperfusion injury: PI3K/Akt pathway involved. Am J Transl Res 9: 5442-5451, 2017.

5. Riquelme I, Tapia O, Leal P, Sandoval A, Varga MG, Letelier $P$, Buchegger K, Bizama C, Espinoza JA, Peek RM, et al: miR-101-2, miR-125b-2 and miR-451a act as potential tumor suppressors in gastric cancer through regulation of the PI3K/AKT/mTOR pathway. Cell Oncol (Dordr) 39: 23-33, 2016.

6. Singh SS, Yap WN, Arfuso F, Kar S, Wang C, Cai W, Dharmarajan AM, SethiG and Kumar AP: Targeting the PI3K/Akt signaling pathway in gastric carcinoma: A reality for personalized medicine? World J Gastroenterol 21: 12261-12273, 2015.

7. Weerink MAS, Struys MMRF, Hannivoort LN, Barends CRM, Absalom AR and Colin P: Clinical pharmacokinetics and pharmacodynamics of dexmedetomidine. Clin Pharmacokinet 56: 893-913, 2017.

8. Hall JE, Uhrich TD, Barney JA, Arain SR and Ebert TJ: Sedative, amnestic, and analgesic properties of small-dose dexmedetomidine infusions. Anesth Analg 90: 699-705, 2000.

9. Lobo FA, Wagemakers M and Absalom AR: Anaesthesia for awake craniotomy. Br J Anaesth 116: 740-744, 2016.

10. Deiner S, Luo X,Lin HM, Sessler DI, Saager L, Sieber FE, Lee HB, Sano M, Jankowski C, Bergese SD, et al; and the Dexlirium Writing Group: Intraoperative infusion of dexmedetomidine for prevention of postoperative delirium and cognitive dysfunction in elderly patients undergoing major elective noncardiac surgery: A randomized clinical trial. JAMA Surg 152: e171505, 2017.

11. Kunnimalaiyaan S, Sokolowski KM, Balamurugan M, Gamblin TC and Kunnimalaiyaan M: Xanthohumol inhibits Notch signaling and induces apoptosis in hepatocellular carcinoma. PLoS One 10: e0127464, 2015.

12. Wu J, Vogel T, Gao X, Lin B, Kulwin C and Chen J: Neuroprotective effect of dexmedetomidine in a murine model of traumatic brain injury. Sci Rep 8: 4935, 2018.
13. Sun YB, Sun SH, Liu DW, Bai B, Zhang YL, Guo YX and Jiang K: The influence of different anesthesia techniques on cognitive dysfunction in elderly patients. Int J Clin Exp Med 9: $16373-16378,2016$

14. Tan WX, Hai N and Mo MZ: The relationship between the serum levels of NSE, S-100 beta protein and IL-6 and postoperative cognitive dysfunction in aged patients with general anesthesia. Guangdong Med 34: 3178-3179, 2013.

15. Li YC, Xi CH, An YF, Dong WH and Zhou M: Perioperative inflammatory response and protein $S-100 \beta$ concentrations relationship with post-operative cognitive dysfunction in elderly patients. Acta Anaesthesiol Scand 56: 595-600, 2012.

16. Hofer S, Steppan J, Wagner T, Funke B, Lichtenstern C, Martin E, Graf BM, Bierhaus A and Weigand MA: Central sympatholytics prolong survival in experimental sepsis. Crit Care 13: R11, 2009.

17. Moriya C, Jinnin M, Yamane K, Maruo K, Muchemwa FC, Igata T, Makino T, Fukushima S and Ihn H: Expression of matrix metalloproteinase-13 is controlled by IL-13 via PI3K/Akt3 and PKC- $\delta$ in normal human dermal fibroblasts. J Invest Dermatol 131: 655-661, 2011.

18. Wang Y, Wu C, Han B, Xu F, Mao M, Guo X and Wang J: Dexmedetomidine attenuates repeated propofol exposureinduced hippocampal apoptosis, PI3K/Akt/Gsk-3 $\beta$ signaling disruption, and juvenile cognitive deficits in neonatal rats. Mol Med Rep 14: 769-775, 2016.

19. Gu J, Sun P, Zhao H, Watts HR, Sanders RD, Terrando N, Xia P, Maze $\mathrm{M}$ and Ma D: Dexmedetomidine provides renoprotection against ischemia-reperfusion injury in mice. Crit Care 15: R153, 2011.

20. Zhu YM, Wang CC, Chen L, Qian LB, Ma LL, Yu J, Zhu MH, Wen CY, Yu LN and Yan M: Both PI3K/Akt and ERK1/2 pathways participate in the protection by dexmedetomidine against transient focal cerebral ischemia/reperfusion injury in rats. Brain Res 1494: 1-8, 2013.

21. Qian XL, Zhang W, Liu MZ, Zhou YB, Zhang JM, Han L, Peng YM, Jiang JH and Wang QD: Dexmedetomidine improves early postoperative cognitive dysfunction in aged mice. Eur J Pharmacol 746: 206-212, 2015.

22. Chen N, Chen X, Xie J, Wu C and Qian J: Dexmedetomidine protects aged rats from postoperative cognitive dysfunction by alleviating hippocampal inflammation. Mol Med Rep 20: 2119-2126, 2019.

This work is licensed under a Creative Commons Attribution-NonCommercial-NoDerivatives 4.0 International (CC BY-NC-ND 4.0) License. 